\title{
Allisartan isoproxil attenuates oxidative stress and inflammation through the SIRT1/Nrf2/NF-кB signalling pathway in diabetic cardiomyopathy rats
}

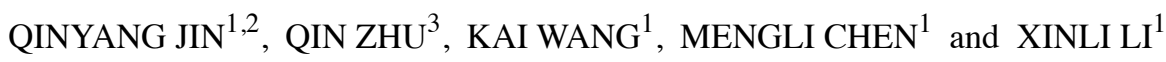 \\ ${ }^{1}$ Department of Cardiology, The First Affiliated Hospital of Nanjing Medical University, Nanjing, Jiangsu 210029; \\ ${ }^{2}$ Department of Cardiology, Zhejiang Provincial People's Hospital, People's Hospital of Hangzhou Medical College, \\ Hangzhou, Zhejiang 310014; ${ }^{3}$ Department of Nephrology, Hangzhou Hospital of Traditional Chinese Medicine, \\ Hangzhou, Zhejiang 310007, P.R. China
}

Received March 12, 2020; Accepted December 22, 2020

DOI: $10.3892 / \mathrm{mmr} .2021 .11854$

\begin{abstract}
Allisartan isoproxil is a new nonpeptide angiotensin II receptor blocker (ARB) precursor drug that is used to treat hypertension and reduce the risk of heart disease. The present study explored the effects of allisartan isoproxil on diabetic cardiomyopathy (DCM) and revealed the roles of hyperglycaemia-induced oxidative stress and inflammation. A rat DCM model was established by high-fat diet feeding in combination with intraperitoneal injection of streptozocin. Echocardiographs showed that diabetic rats exhibited significantly decreased cardiac function. Troponin $\mathrm{T}$ (cTnT) and B-type natriuretic peptide (BNP) were significantly increased in DCM rats as obtained by ELISA. Allisartan isoproxil significantly improved the EF\% and E'/A' ratio. Histopathologic staining showed that allisartan isoproxil prevented histological alterations, attenuated the accumulation of collagen, and ameliorated cTnT and BNP levels. Western blot and immunohistochemical results indicated that the expression levels of silent information regulator 2 homologue 1 (SIRT1) and nuclear factor erythroid 2-related factor 2 (Nrf2) were decreased in the hearts of diabetic rats, and antioxidant defences were also decreased. In addition, allisartan isoproxil decreased the expression of NF- $\mathrm{B}$ p 65 and the inflammatory cytokines TNF- $\alpha$ and IL- $1 \beta$ which were determined by reverse transcription-quantitative PCR in the diabetic heart. Western blotting and TUNEL staining results also showed that cardiac Bax and cleaved caspase- 3 and the number of apoptotic myocardial cells were increased
\end{abstract}

Correspondence to: Professor Xinli Li, Department of Cardiology, The First Affiliated Hospital of Nanjing Medical University, 300 Guangzhou Road, Nanjing, Jiangsu 210029, P.R. China E-mail: medfuture@sina.com

Key words: diabetic cardiomyopathy, allisartan isoproxil, inflammation, oxidative stress, silent information regulator 2 homologue 1, nuclear factor erythroid 2-related factor 2, NF- $\kappa B$ in the diabetic heart and decreased following treatment with allisartan isoproxil. In conclusion, the present results indicated that allisartan isoproxil alleviated DCM by attenuating diabetes-induced oxidative stress and inflammation through the SIRT1/Nrf2/NF- $\kappa$ B signalling pathway.

\section{Introduction}

Diabetes is one of the major diseases threatening human health worldwide. According to official reports, more than 400 million people worldwide suffer from diabetes (1). Diabetic cardiomyopathy (DCM) is one of the complications of diabetes; moreover, it is one of the main causes of diabetes-associated morbidity and mortality. Furthermore, DMC accounts for more than $50 \%$ of all diabetes-associated deaths each year (2). According to epidemiological studies, people with diabetes are 2 to 5 times more likely to develop heart failure than their healthy peers. Therefore, it is urgent to explore new prevention and treatment strategies for DCM (3-5). Complex biological responses, including inflammation, oxidative stress, the renin-angiotensin system, mitochondrial dysfunction, intracellular $\mathrm{Ca}_{2}{ }^{+}$transport disorders, myocardial fibrosis, and cardiomyocyte apoptosis, are associated with DCM, which makes it extremely difficult to investigate the pathophysiological mechanism of DCM $(6,7)$.

A growing body of evidence has confirmed that oxidative stress, inflammation, and cell death play major roles in DCM $(6,8,9)$. Both hyperglycaemia and inflammation can lead to excessive reactive oxygen species (ROS) production in cells, which can cause lipid peroxidation reactions and reduce the antioxidant capacity of cells, ultimately leading to cardiomyocyte apoptosis and even the occurrence of cardiac dysfunction (10). In addition, ROS and ROS-induced DNA damage can promote inflammatory responses and fibrotic processes (11).

Nuclear factor erythroid 2-related factor $2(\mathrm{Nrf} 2)$ is an important redox sensor and one of the key regulators of the expression of various antioxidants in cells $(12,13)$. In response to oxidative stress, Nrf2 is activated and binds to antioxidant response elements (AREs) to activate the transcription 
and translation of antioxidant genes and proteins, respectively $(14,15)$. Zhao et al (16) reported that the inhibition of $\mathrm{Nrf} 2$ expression affected the expression level of antioxidant proteins, thus exacerbating DOX-induced oxidative stress damage in cardiomyocytes. Silent information regulator 2 homologue 1 (SIRT1) is an $\mathrm{NAD}^{+}$-dependent deacetylase that can regulate a variety of biological processes, including cell metabolism, redox homeostasis, apoptosis, inflammation, and senescence, through deacetylation $(17,18)$. Existing research has confirmed that SIRT1 suppresses cardiomyocyte apoptosis in DCM (18). Moreover, in a study of glomerular mesangial cells, SIRT1 was revealed to activate the Nrf2/ARE pathway (19). Nuclear factor $-\kappa \mathrm{B}(\mathrm{NF}-\kappa \mathrm{B})$ is a key transcription factor in the regulation of inflammatory processes, and SIRT1 can also modulate $\mathrm{NF}-\kappa \mathrm{B}$-dependent inflammatory responses (20). Therefore, it is critical to explore whether the SIRT1/Nrf2/NF- $\kappa \mathrm{B}$ signalling pathway plays a regulatory role in oxidative stress and inflammation in diabetic cardiomyopathy.

Hyperglycaemia activates the renin-angiotensin (Ang) system (RAS) and induces extracellular matrix accumulation, leading to cardiac remodelling and dysfunction (21). Briefly, increased levels of Ang II have been reported to regulate the production of ROS and lead to cardiac hypertrophy, left ventricular dysfunction, and myocardial insulin resistance $(22,23)$. Allisartan isoproxil is a new nonpeptide angiotensin II receptor blocker (ARB) precursor drug that can produce a carboxylic acid derivative (EXP3174) during absorption in vivo $(24,25)$. Compared with losartan, allisartan isoproxil can be completely hydrolysed by esterase into the active metabolite EXP3174 after being absorbed into the small intestine and stomach (26). Losartan is already widely used to treat hypertension clinically (27), and the effect of losartan has been investigated in ventricular hypertrophy, heart failure, kidney diseases and DCM in rats (28-32). However, it remains to be seen whether allisartan isoproxil can be used for the treatment of DCM.

In the present study, it was examined whether allisartan isoproxil protected against cardiac injuries in DCM rats. The present study investigated the antioxidant stress and anti-inflammatory effects of allisartan isoproxil in myocardium by constructing DCM rats models and treating them with allisartan isoproxil. The experiments focused on the regulatory effect of allisartan isoproxil on SIRT1/Nrf2 and SIRT1/NF- $\kappa$ B signaling pathways. The results indicated that allisartan isoproxil could significantly attenuate cardiac injuries caused by diabetes by inhibiting inflammation and oxidative stress.

\section{Materials and methods}

Animal model of diabetes. Wild-type SD rats were obtained from Shanghai Sippe-Bk Lab Animal Co., Ltd. All animal study protocols were approved by the Institutional Ethics Committee of The First Affiliated Hospital with Nanjing Medical University (approval no. IACUC-1803019) and were performed according to the guidelines of the US Department of Health (NIH Publication no. 85-23, revised 1996) for the use and care of laboratory animals.

A rat diabetes model was established by streptozocin (STZ) and a high-fat diet (HFD) (33). The HFD (10\% lard oil, 10\% sucrose, $2.0 \%$ cholesterol, $0.5 \%$ cholate, $5 \%$ yolk powder and $72.5 \%$ ordinary feed) and control diets were obtained from Beijing Boaigang Biological Technology Co., Ltd. A total of 32 adult Sprague-Dawley rats weighing 280-300 g were used for the animal experiments. The animals were housed with a 12-h light/dark cycle and a temperature of $22 \pm 2^{\circ} \mathrm{C}$. Rats in the control group were fed a control diet. The other 3 groups were fed an HFD to induce diabetes. After 4 weeks of feeding, all the animals were fasted for $12 \mathrm{~h}$, and then the rats in the diabetes group were intraperitoneally injected with STZ $(35 \mathrm{mg} / \mathrm{kg}$ body weight), while the rats in the control group were injected with the same volume of citrate buffer (34-36). The blood glucose levels were measured $72 \mathrm{~h}$ after STZ injection. When the blood glucose levels were higher than $16.7 \mathrm{mmol} / 1$, the rats were identified as diabetic. Then, the rats were continued on an HFD for 8 weeks to induce DCM. To explore the effects of allisartan isoproxil, after 8 weeks of continuous HFD feeding, the 3 groups of diabetic rats were treated with PBS (DCM+PBS group), allisartan isoproxil (10.8 mg/kg/day, DCM+allisartan isoproxil group) $(24,37)$, and no treatment other than an HFD (DCM group). In the present study, the allisartan isoproxil was used as palliative treatment. The rats were fed an HFD throughout the 16-week experimental period. The whole experiment lasted for 16 weeks. During the experimental period, the rats were monitored every day, and 4 of them exhibited lethargy after STZ injection and succumbed to natural causes within a week. At the end of the experiment period, 28 rats were anesthetized with isoflurane (2.0-2.5\% isoflurane in oxygen). When the rats appeared to have faint breathing, myasthenia, lack of independent reaction, cyanosis, or were comatose, it was considered that rats were close to death and were euthanized by cervical dislocation.

Echocardiography. A Vevo 2100 imaging system (VisualSonics, Inc.) was used to measure heart function in all animals by 2D echocardiography as previously described (7). Left ventricular ejection fraction (EF), short axis fractional shortening (FS), the E'/A' ratio, the left ventricular end-systolic dimension (LVD) and left ventricular posterior wall thickness (LVPW) were measured from the parasternal long-axis view.

Histological analysis. Heart tissues were fixed in $10 \%$ neutral buffered formalin for $72 \mathrm{~h}$ at room temperature. Histopathological analysis was performed on paraffin-embedded sections of the heart $(5 \mu \mathrm{m})$ stained with hematoxylin and eosin staining. Briefly, after dewaxing and hydration of paraffin sections, hematoxylin staining was performed for $3 \mathrm{~min}$ at room temperature, the sections rinsed with distilled water, and then stained with eosin for 2 min at room temperature and rinsed with distilled water again. Finally, the sections were dried and sealed. Myocardial fibrosis was determined using Masson's trichrome staining (Sigma-Aldrich; Merck KGaA). Briefly, hematoxylin staining for $5 \mathrm{~min}$, ponceau trichrome staining for $10 \mathrm{~min}$ and aniline blue staining for $2 \mathrm{~min}$ at room temperature. Finally, the sections were dried and sealed. Multiple images were acquired from each stained heart section under a light microscope. The fibrotic areas of paraffin-embedded heart sections were evaluated using ImageJ 6.0 software (National Institutes of Health). 
Enzyme-linked immunosorbent assay. Serum cTnT and BNP levels were measured by ELISA kits (cat. nos. OKEH04575 and OKEH00475; Aviva Systems Biology) according to the manufacturer's instructions. Briefly, serum samples were added to an ELISA plate at a standard volume of $100 \mu \mathrm{l} /$ well. The plates were incubated at $37^{\circ} \mathrm{C}$ for $2 \mathrm{~h}$. Then, the plates were washed, the conjugation solution was added to the wells and incubated at $37^{\circ} \mathrm{C}$ for another $1 \mathrm{~h}$. Finally, the results were recorded using a microplate reader (Elx800; BioTek Instruments, Inc.) at a wavelength of $450 \mathrm{~nm}$.

Immunohistochemistry. Heart tissues were fixed in $10 \%$ neutral buffered formalin for $72 \mathrm{~h}$ at room temperature. The expression of SIRT1 and Nrf2 in cardiac tissue was examined by immunohistochemistry. Paraffin-embedded sections $(5 \mu \mathrm{m})$ were deparaffinized (heated in a drying oven at $60^{\circ} \mathrm{C}$ for $2 \mathrm{~h}$ and soaked in pure xylene for 15 min three times) and hydrated (immersion in 100, 90, 85, and 75\% alcohol, $10 \mathrm{~min}$ each). Then, the activity of endogenous peroxidase was quenched with $3 \% \mathrm{H}_{2} \mathrm{O}_{2}$ for $20 \mathrm{~min}$. After endogenous peroxidase quenching and antigen retrieval, to avoid nonspecific antibody binding, the sections were placed in a culture dish containing $10 \%$ goat serum (cat. no. ab7481; Abcam) and incubated for $30 \mathrm{~min}$ at room temperature. Then, the sections were incubated with SIRT1 (1:400, cat. no. ab189494) or Nrf2 (1:400, cat. no. ab92946; both from Abcam) antibodies at $4^{\circ} \mathrm{C}$ for $12 \mathrm{~h}$. Following primary antibody incubation, the sections were washed with PBS three times. The sections were then incubated in a diluted solution of horseradish peroxidase-conjugated goat anti-rabbit secondary antibody (1:50, cat. no. ab205718; Abcam) for 60 min. 3,3-diaminobenzidine was used as the detection reagent for $5 \mathrm{~min}$. Finally, the sections were counterstained with haematoxylin (1 $\mathrm{min}$ at room temperature) and bluing reagent, dehydrated and mounted. Multiple images were acquired from each stained heart section under a light microscope. The heart sections were evaluated using ImageJ 6.0 software (National Institutes of Health).

TUNEL assay. Rat heart tissue was surgically removed, frozen in optima cutting temperature compound and sectioned at $8 \mu \mathrm{m}$ with a cryostat. Apoptosis in rat heart tissue was measured using the ApopTag plus peroxidase in situ apoptosis detection kit (Sigma-Aldrich; Merck KGaA) according to the manufacturer's guidelines. Briefly, after washing with PBS, heart frozen sections were incubated with $1 \mu \mathrm{g} / \mathrm{ml}$ Proteinase $\mathrm{K}$ and $10 \mathrm{mM}$ Tris solution for $10 \mathrm{~min}$ at room temperature. The sections were washed in PBS for 5 min twice and then incubated with the TUNEL reaction mixture at room temperature for $45 \mathrm{~min}$. Finally, frozen sections were washed with PBS 5 min twice and then the nuclei labeled with DAPI for $20 \mathrm{~min}$ at room temperature and mounted in $50 \%$ glycerine diluted in water. For each rat, three sections were used for the TUNEL assay. A total of 15 randomly chosen microscopic fields were analyzed under a fluorescence microscope. The number of TUNEL-positive myocardial nuclei and total myocardial nuclei in each group were calculated. Images were captured with a fluorescent microscope. The rate of apoptosis was determined by dividing the number of TUNEL-positive myocardial nuclei by the total number of myocardial nuclei.
Measurement of malondialdehyde (MDA) production and superoxide dismutase (SOD) activity. Experimental kits were used to determine the level of MDA (cat. no. YBA003-2) and SOD (cat. no. YBA001-3) activity in myocardial tissue according to the manufacturer's instructions (Shanghai Yubo Biological Technology Co., Ltd.).

Reverse transcription-quantitative $(R T-q) P C R$. Total RNA was extracted from heart tissue with TRIzol reagent (CWBiotech). To reverse transcribe RNA into cDNA for RT-qPCR, TransStart Tip Green qPCR SuperMix from TransGen Biotech was used according to the manufacturer's instructions. Quantitative PCR was performed using SYBR green (Thermo Fisher Scientific, Inc.). Thermocycling conditions were $2 \mathrm{~min}$ at $50^{\circ} \mathrm{C}$, followed by initial denaturation at $95^{\circ} \mathrm{C}$ for $2 \mathrm{~min}$ and 45 cycles at $95^{\circ} \mathrm{C}$ for $15 \mathrm{sec}$ (denaturation) and $60 \mathrm{sec}$ at $56^{\circ} \mathrm{C}$ (annealing and extension). The relative expression level was calculated using the $2^{-\Delta \Delta \mathrm{Cq}}$ method (38). The primers are listed in Table SI.

Western blotting. Total protein was extracted from the samples using radioimmunoprecipitation assay (RIPA) lysis buffer $(100 \mu \mathrm{l})$. The lysates were centrifuged at $12,000 \mathrm{x} \mathrm{g}$ for $20 \mathrm{~min}$ at $4^{\circ} \mathrm{C}$ and the supernatants collected. The protein concentration was determined by the BCA assay. Protein samples $(20 \mu \mathrm{g})$ were loaded and separated using 8 or $10 \%$ sodium dodecyl sulfate (SDS)-polyacrylamide gel electrophoresis at $90 \mathrm{~V}$ for $1.5 \mathrm{~h}$ and subsequently transferred to polyvinylidene fluoride membranes. The membrane was blocked in 5\% bovine serum albumin (cat. no. A3858, Sigma-Aldrich) for $2 \mathrm{~h}$ at room temperature. The primary antibodies used were SIRT1 (1:1,000; cat. no. cat. no. ab189494), Nrf2 (1:1,000; cat. no. ab92946), heme oxygenase-1 (HO-1; 1:1,000; cat. no. ab13243), NF-кB p65 (1:1,000; cat. no. ab16502), TNF- $\alpha$ (1:1,000; cat. no. ab66579), IL-1 $\beta$ (1:1,000; cat. no. ab9722), Bax (1:1,000; cat. no. ab32503), Bcl-2 (1:1,000; cat. no. ab196495), caspase-3 (1:1,000; cat. no. ab4051), cleaved caspase-3 (1:1,000; cat. no. ab49822), and GAPDH (1:2,000; cat. no. ab9485; all from Abcam). Following an overnight incubation at $4{ }^{\circ} \mathrm{C}$ the membranes were washed with TBST three times, and then horseradish peroxidase (HRP)-conjugated rabbit IgG secondary antibody (1:5,000; cat no. sc-2004; Santa Cruz Biotechnology, Inc.) were added for each corresponding primary antibody for $1.5 \mathrm{~h}$ at room temperature. All protein bands were visualized by enhanced chemiluminescence (ECL) system using the ECL chemiluminescent substrate reagent kit (cat. no. C510043 Thermo Fisher Scientific, Inc.) according to the manufacturer's protocol. The grayscale value of protein bands was calculated using Image J 6.0 software.

Statistical analysis. The data are expressed as the mean \pm SD of at least three independent experiments for each in vitro experimental group and at least six independent experiments for each animal group. All experimental data were analysed using SPSS (version 20.0; IBM Corp.) and GraphPad Prism 7.0 (GraphPad Software, Inc.) unless otherwise stated. The data was evaluated with unpaired Student's t-tests for comparisons between two groups. A value of $\mathrm{P}<0.05$ was considered to indicate a statistically significant result. 
A

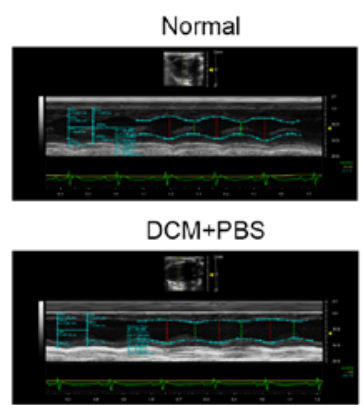

C

E
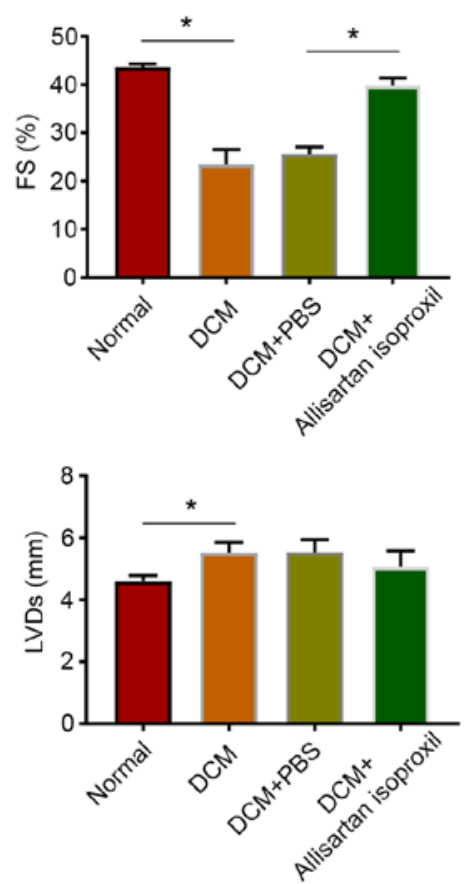

B
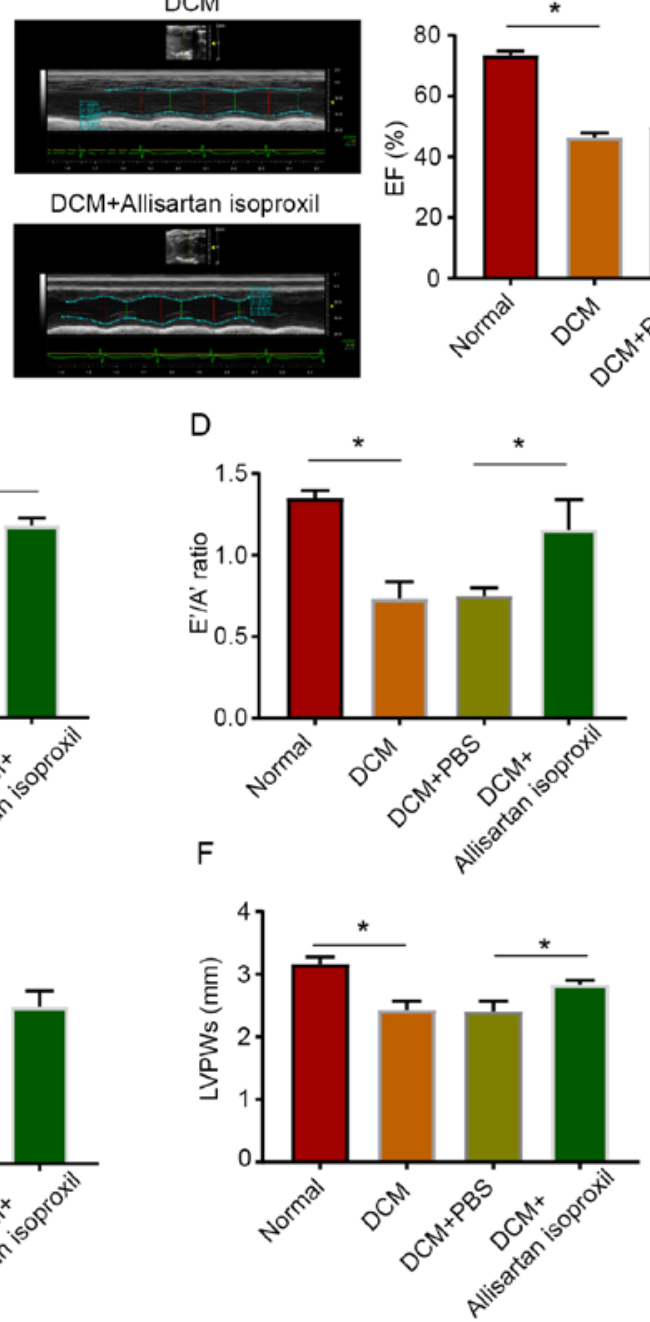

Figure 1. Protective effects of allisartan isoproxil on the hearts of DCM rats. (A) Representative echocardiography images. Twelve weeks after diabetes induction, the rats were characterized as having decreased cardiac function. (B) Reduced EF (\%). (C) Left ventricular FS (\%). (D) The ratio of the early (E) to late (A) ventricular filling velocities (E'/A' ratio). (E) LVD and (F) LVPW at end-systole. The values are the mean \pm SD of $n=6$ in each group. *P<0.05. DCM, diabetic cardiomyopathy; EF, ejection fraction; FS, fractional shortening; LVD, left ventricular end-systolic dimension; LVPW, left ventricular posterior wall thickness.

\section{Results}

Protective effects of allisartan isoproxil on the hearts of DCM rats. To investigate the effect of allisartan isoproxil on cardiac function in diabetic rats, cardiac function was measured by echocardiography in diabetic rats treated with and without allisartan isoproxil (Fig. 1A). As revealed in Fig. 1, the diabetic rats exhibited abnormal EFs and FS dysfunction (Fig. 1B and C). Moreover, cardiac diastolic dysfunction with a reduced $\mathrm{E}^{\prime} / \mathrm{A}^{\prime}$ ratio was also detected in diabetic rats (Fig. 1D). In addition, both the LVD and LVPW exhibited abnormal manifestations (Fig. 1E and F). However, cardiac systolic function and cardiac diastolic function were significantly improved by allisartan isoproxil (Fig. 1A-F).

Histological and cardiac marker analysis of DCM rats. Next, the myocardial damage caused by hyperglycaemia and the protective effects of allisartan isoproxil were explored. The haematoxylin-stained histopathological sections of normal control rats exhibited normal myocardial tissue morphology and regular nuclear arrangement. However, STZ-induced
DCM rats exhibited tissue oedema, inflammatory cell infiltration and disorganized myocardial nuclei (arrowhead), while allisartan isoproxil treatment significantly alleviated these pathological effects (Fig. 2A). Moreover, allisartan isoproxil significantly attenuated the accumulation of collagen (arrowhead) in the myocardial tissue of DCM rats, as revealed by Masson staining (Fig. 2B). Then, cardiac troponin I (cTnI) and B-type natriuretic peptide (BNP), biomarkers of myocardial injury and heart failure, respectively, were measured by ELISA. Allisartan isoproxil effectively inhibited the expression levels of both cTnT and BNP (Fig. 2C and D).

Allisartan isoproxil can inhibit diabetes-induced myocardial oxidative stress in rats. It has been confirmed that SIRT1 and Nrf 2 could reduce myocardial cell apoptosis and modulate the antioxidant response system in DCM (18). Nrf2 has been reported to modulate the antioxidant response system of cardiomyocytes under high glucose conditions (39). When $\mathrm{Nrf} 2$ is activated, it can enter the nucleus and regulate the expression level of the antioxidative stress response gene HO-1 to protect cell viability $(13,39)$. Moreover, a study on 

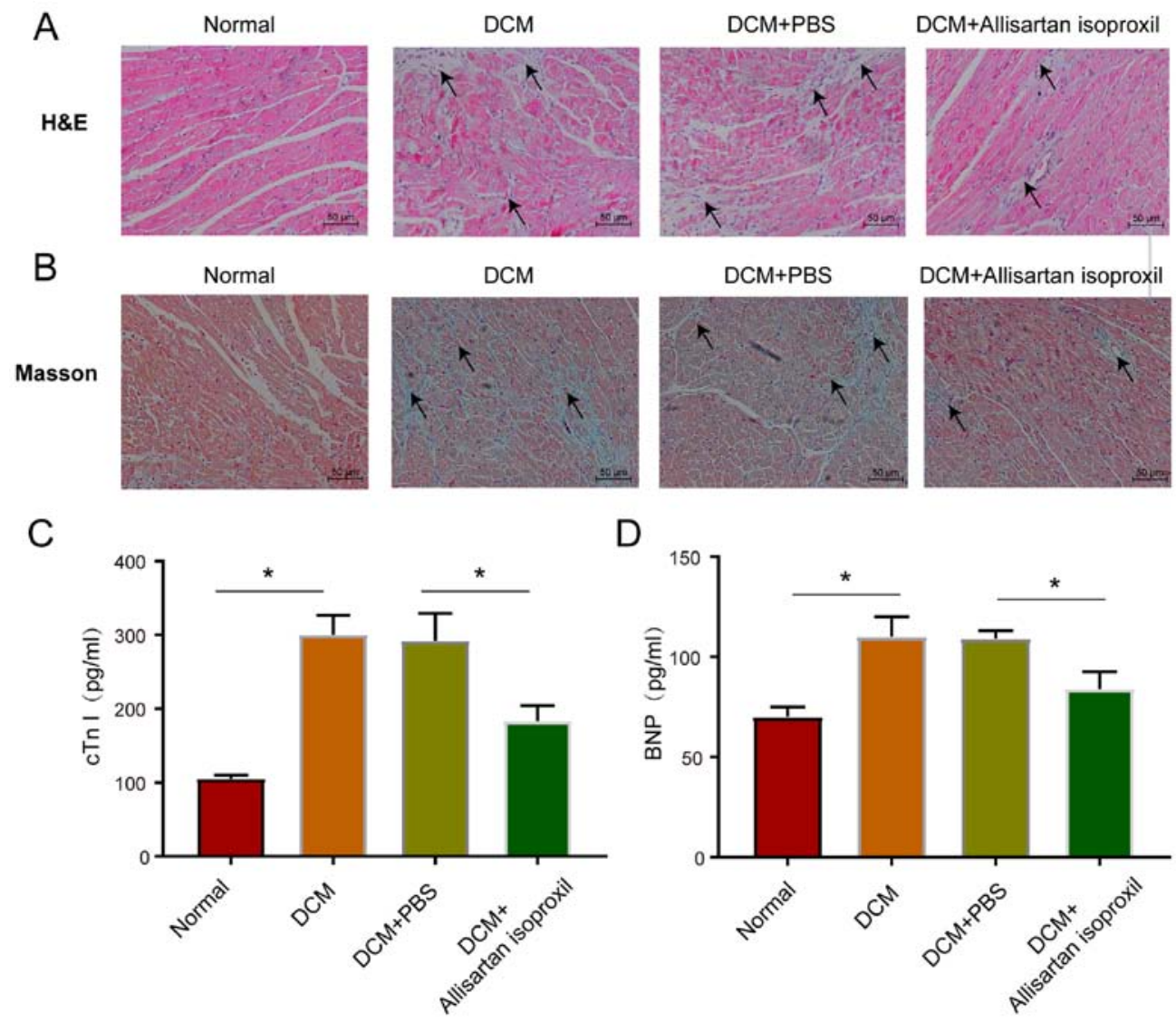

Figure 2. Histological and cardiac marker analysis of DCM rats. (A and B) Staining of heart tissues harvested at the end of the treatment period. (A) Representative images of myocardial tissue sections stained with H\&E; scale bar, $50 \mu \mathrm{m}$. Inflammatory cell infiltration and disorganized myocardial nuclei (arrowhead). (B) Assessment of cardiac fibrosis by Masson's trichrome staining; scale bar, $50 \mu \mathrm{m}$. The accumulation of collagen (arrowhead). (C) Serum levels of cTnI. (D) Serum levels of BNP. The values are the mean $\pm \mathrm{SD}$ of $\mathrm{n}=6$ in each group. ${ }^{*} \mathrm{P}<0.05$. DCM, diabetic cardiomyopathy; cTnI, cardiac troponin I; BNP, B-type natriuretic peptide.

glomerular mesangial cells revealed that SIRT1 could activate the Nrf2/ARE pathway (19). It was hypothesised that SIRT1 could regulate DCM by regulating the expression of Nrf2. Therefore, western blotting and RT-qPCR were used to assess the protein and mRNA expression levels, respectively, of SIRT1 and Nrf2. As revealed in Fig. 3A and C, the protein and mRNA expression levels of SIRT1 and Nrf 2 in the myocardial tissue of diabetic rats were significantly downregulated, while allisartan isoproxil treatment partially restored the expression levels of SIRT1 and Nrf2 (Fig. 3A). Immunohistochemical staining also revealed that allisartan isoproxil could reverse the downregulation of both SIRT1 and Nrf2 protein levels in the heart tissue sections of diabetic rats (Fig. 3B). Moreover, as was anticipated, the expression trend of $\mathrm{HO}-1$, a downstream gene of $\mathrm{Nrf2}$, was consistent with that of Nrf2 (Fig. 3C). The expression level of MDA (Fig. 3D) and the activity of SOD) (Fig. 3E were also assessed. These results revealed that allisartan isoproxil could reduce the oxidative stress response of DCM rats.

Allisartan isoproxil can attenuate inflammation in DCM rats. As a transcription factor, NF- $\kappa \mathrm{B}$ is activated by phosphorylation and transfers into the nucleus to regulate the transcription of inflammatory cytokine genes, including TNF- $\alpha$ and IL-1 $\beta(40,41)$. The protein level of phosphory-

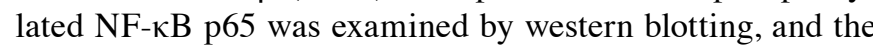
mRNA level was measured by RT-qPCR. The present data indicated that the protein level of NF- $\kappa \mathrm{B}$ p 65 was significantly upregulated in DCM rats, while allisartan isoproxil treatment partially restored the protein and mRNA levels of $N F-\kappa B$ p65 (Fig. 4A and B). In addition, allisartan isoproxil significantly reduced the upregulation of TNF- $\alpha$ and IL- $1 \beta$ protein expression in DCM rats (Fig. 4D). Consistent results were also obtained by RT-qPCR (Fig. 4C).

\section{Cardiomyocyte apoptosis in DCM rats is reduced by allisartan} isoproxil. Myocardial cell injury leads to cell apoptosis (6). To investigate the protective effect of allisartan isoproxil on myocardial apoptosis in DCM rats, the expression levels of Bax, Bcl-2, pro-caspase-3 and cleaved caspase-3 were assessed by western blotting (Fig. 5A). The Bax/Bcl-2 and cleaved caspase-3/pro-caspase-3 ratios were significantly decreased in DCM rats treated with allisartan isoproxil (Fig. 5A). Moreover, cardiomyocytes underwent apoptotic death in diabetic cardiomyopathy, as evidenced by increased positive TUNEL staining, but this effect was reversed by allisartan isoproxil (Fig. 5B). In summary, the decrease in proapoptotic proteins, the increase in antiapoptotic proteins and the decrease in apoptotic cardiomyocytes all indicated that allisartan isoproxil protected cardiomyocytes from apoptosis in DCM.

\section{Discussion}

Diabetes is known to cause numerous complications, including cardiomyopathy. Persistent high blood glucose levels cause 
A

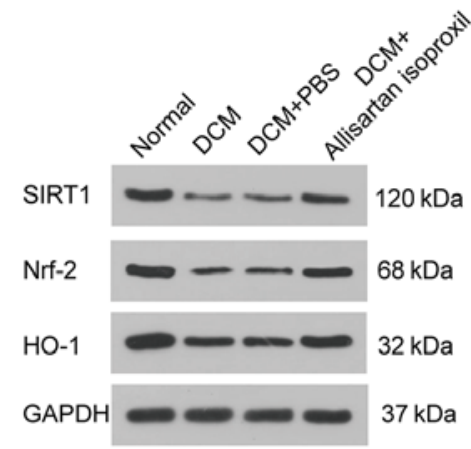

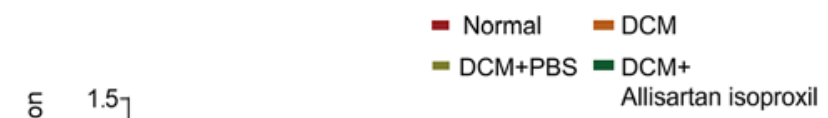

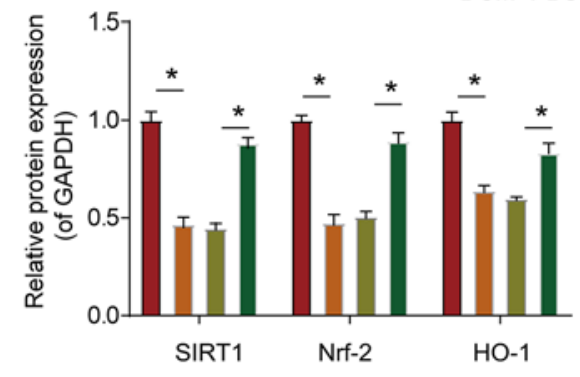

B
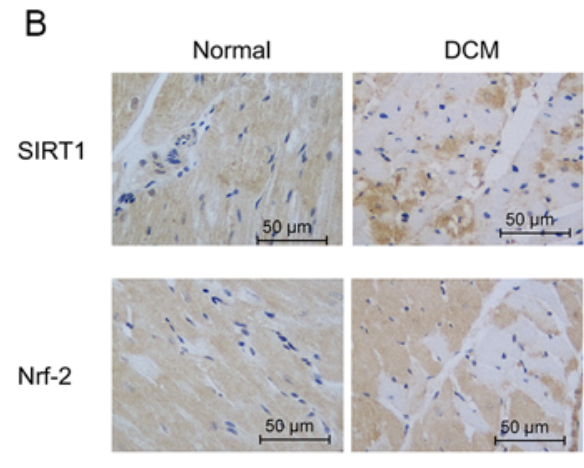

C

D

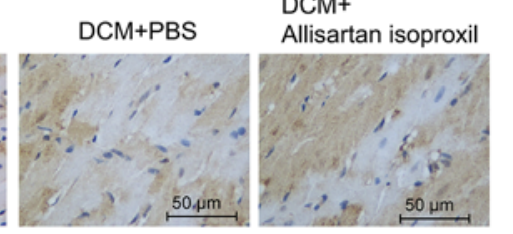

$\begin{aligned}- \text { Normal } & =\mathrm{DCM} \\ =\mathrm{DCM}+\mathrm{PBS} & =\mathrm{DCM}+ \\ & \text { Allisartan isoproxil }\end{aligned}$
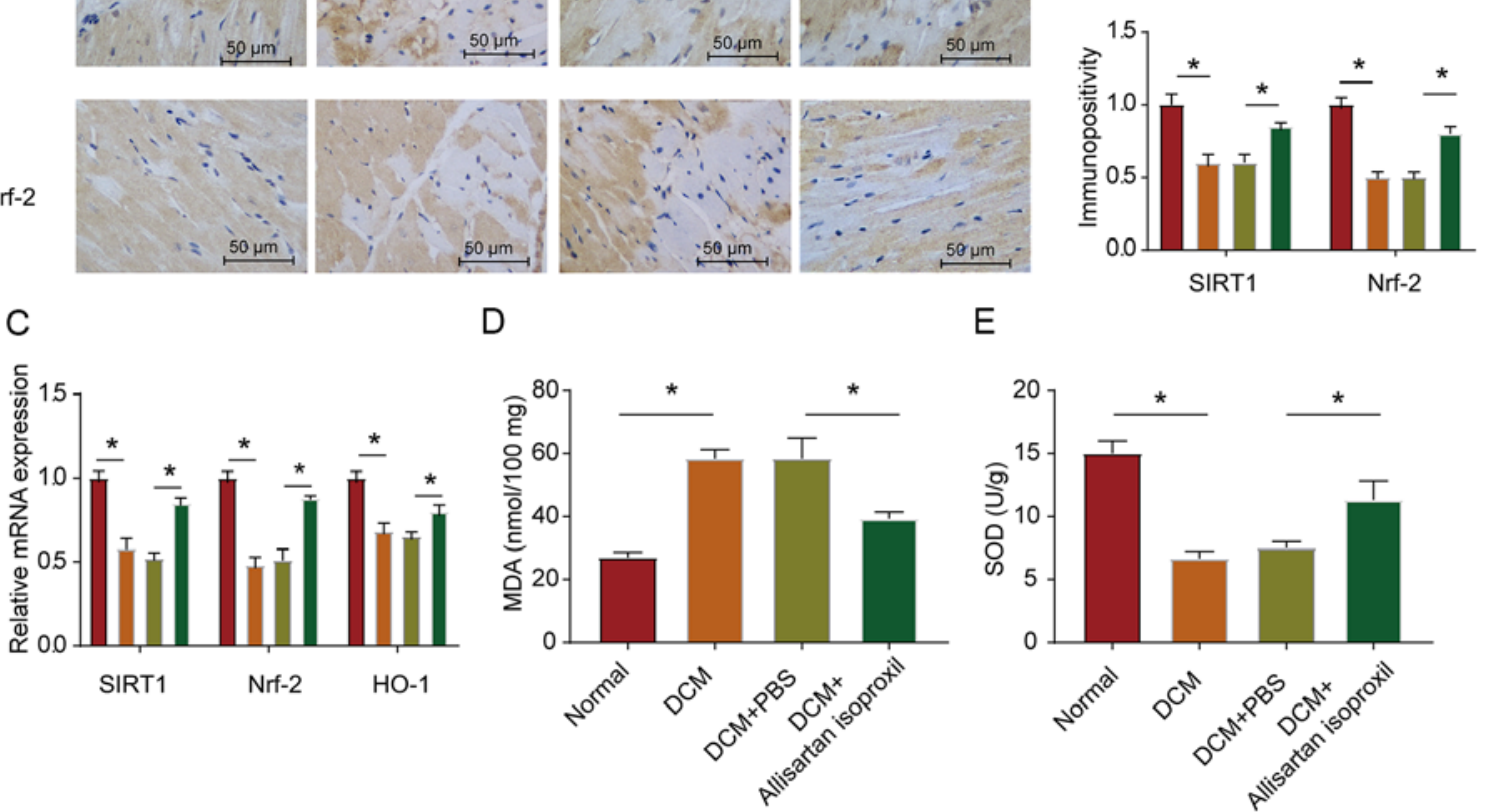

E

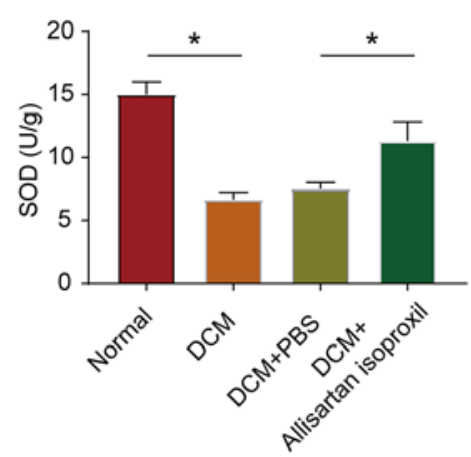

Figure 3. Allisartan isoproxil can inhibit diabetes-induced myocardial oxidative stress in rats. (A) Western blot analysis of SIRT1, Nrf2 and HO-1 expression in lysates prepared from heart tissues. The myocardial tissues were subjected to immunohistochemical analysis as described in the Materials and methods. (B) Representative images were acquired and quantified for SIRT1 and Nrf2. (C) The expression levels of SIRT1, Nrf2 and HO-1 were determined by reverse transcription-quantitative PCR, and the grouping was the same as in B. (D and E) Oxidative stress in myocardial tissues was determined by measuring the levels of (D) MDA and (E) SOD in lysates prepared from heart tissues, and the grouping was the same as in B. The values are the mean \pm SD of $n=6$ in each group. * $\mathrm{P}<0.05$. SIRT1, silent information regulator 2 homologue 1; Nrf2, nuclear factor erythroid 2-related factor 2; HO-1, heme oxygenase-1; MDA, malondialdehyde; SOD, superoxide dismutase; DCM, diabetic cardiomyopathy.

oxidative stress, inflammation, and apoptosis, and these pathological reactions are related to the pathogenesis of DCM $(42,43)$. Long-term diabetes is associated with persistently high blood glucose levels and may be associated with myocardial tissue damage. The exacerbation of oxidative stress injury and increased inflammatory response under sustained hyperglycaemia are considered to be the key mechanisms of DCM induction $(6,8)$. These two harmful mechanisms promote myocardial tissue fibrosis, activate the apoptosis pathway, and ultimately damage the structure and function of the heart $(44,45)$. Allisartan isoproxil is a new nonpeptide angiotensin II receptor blocker precursor drug. Compared with losartan, allisartan isoproxil has higher absorption efficiency, better blood pressure lowering effect and lower adverse reactions $(25,26)$. In the present study, it was revealed that treatment of DCM with allisartan isoproxil in diabetic rats alleviated inflammation and oxidative stress injury. The protective effect of allisartan ester was achieved by inhibiting the aberrant activation of $\mathrm{NF}-\kappa \mathrm{B}$ and activating the antioxidant $\mathrm{Nrf} 2$ system. However, it should be noted that when designing the experiment, we neglected to set a positive reference group. Nonetheless, our results provide insights into the future exploration of allisartan isoproxil in the treatment of DCM.

A rat model of diabetes induced by STZ and an HFD was first established. The effect of allisartan isoproxil on cardiac function in diabetic rats was investigated by echocardiography, and the results revealed that diabetic rats exhibited cardiac dysfunction. However, allisartan isoproxil protected the heart functions of the rats by increasing their heart EF\%, E'/A' ratio and LVPWs. Notably, LVPWs in our diabetic rat model was reduced, which is consistent with the results of Zhao et al (8). Histological examination of the diabetic heart revealed 
A

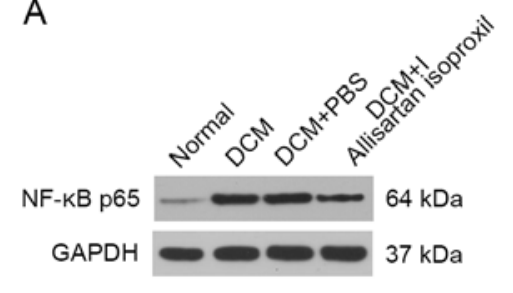

C

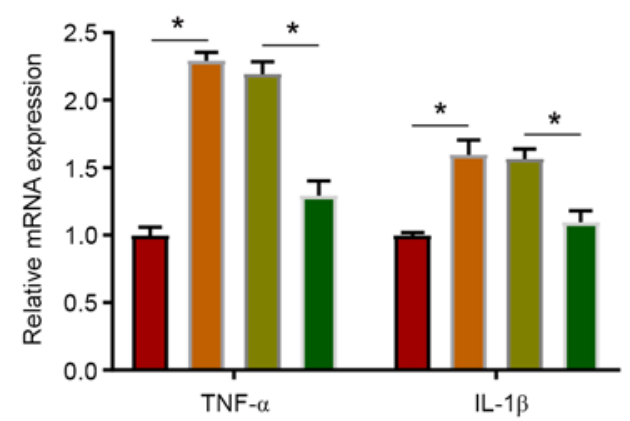

D

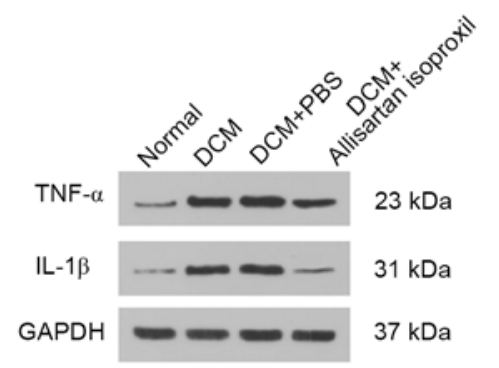

B
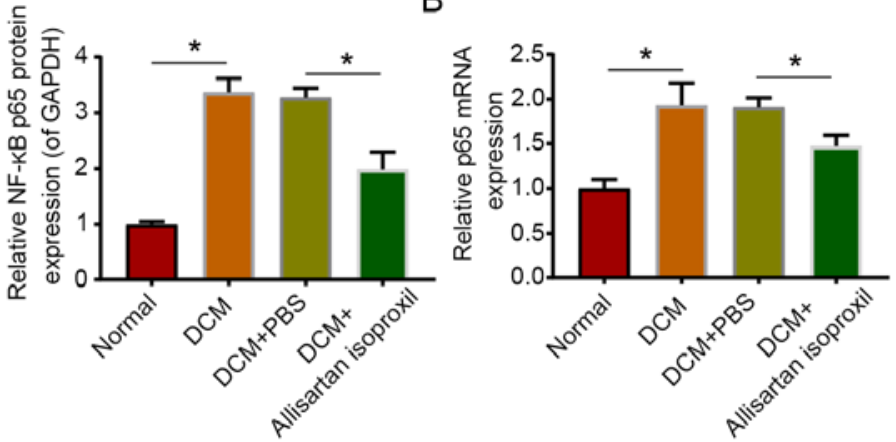
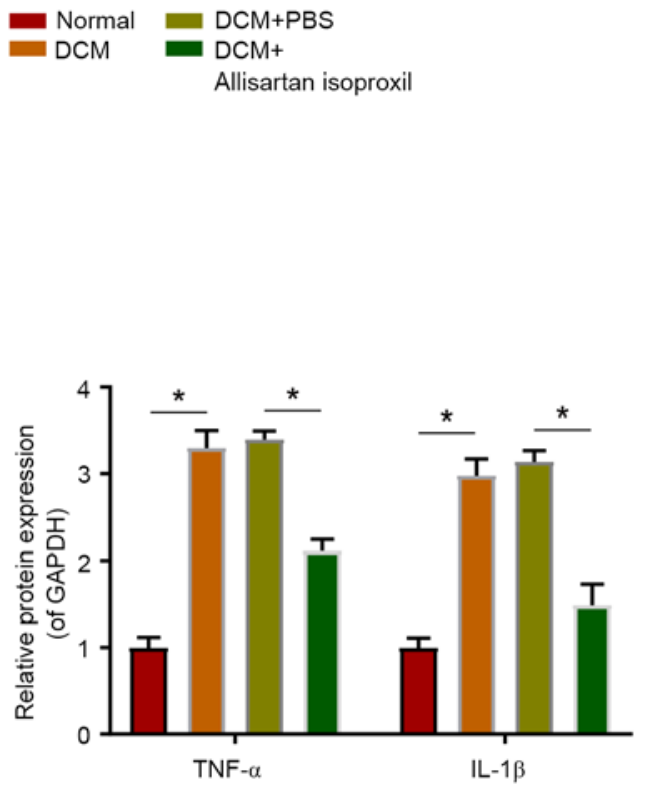

Figure 4. Allisartan isoproxil can attenuate inflammation in DCM rats. (A and B) The protein and mRNA expression levels of NF- $\kappa$ B were measured by western blotting and reverse transcription-quantitative PCR, respectively. (C and D) The inflammatory response in the myocardial tissues was determined by measuring the protein and mRNA expression levels of TNF- $\alpha$ and IL-1 $\beta$. The values are the mean \pm SD of $n=6$ in each group. * $\mathrm{P}<0.05$. DCM, diabetic cardiomyopathy; TNF- $\alpha$, tumour necrosis factor- $\alpha$; IL-1 $\beta$, interleukin- $1 \beta$.

increased myocardial damage and collagen deposition (33), consistent with the present results. Furthermore, aberrant expression of apoptosis-related proteins was also revealed in the heart tissues of DCM rats. Hyperglycaemia-induced oxidative stress, inflammation and cell damage are the main causes of myocardial fibrosis $(46,47)$.

$\mathrm{NF}-\kappa \mathrm{B}$, a key transcription factor associated with inflammatory responses, activates $\mathrm{NF}-\kappa \mathrm{B}$ translocation into the nucleus and regulates the expression of related genes, including TNF- $\alpha$, IL-1 $\beta$ and IL-6 $(48,49)$. Moreover, NF- $\kappa \mathrm{B}$ has been revealed to play a pivotal role in the development of DCM (50). A recent study reported that activation of $\mathrm{NF}-\kappa \mathrm{B}$ induced increased oxidative stress, which could lead to heart dysfunction in DCM (51). Notably, inflammatory pathways in DCM are closely related to oxidative stress signalling and ultimately promote the development of myocardial injury $(52,53)$. In the present study, DCM was associated with increased cardiac NF- $\mathrm{B}$ p 65 and TNF- $\alpha$ expression, while allisartan isoproxil treatment partially restored the protein and mRNA levels of NF- $\kappa$ B p65 and IL-1 $\beta$. Allisartan isoproxil treatment prevented cardiac injury in diabetic rats, and this effect may be attributed to attenuated inflammation.

A growing body of evidence has revealed that oxidative stress caused by excessive ROS in hyperglycaemia is associated with the progression of myocardial injury $(54,55)$. Cardiac oxidative stress is closely related to myocardial fibrosis and cardiomyocyte apoptosis, and excessive oxidative stress can lead to severe cardiac dysfunction and heart failure $(8,11)$. $\mathrm{Nrf2}$, a member of the nuclear factor erythroid 2 family of nuclear basic leucine-zipper transcription factors, activates the transcription and translation of several antioxidant genes and proteins by binding to AREs (19). As one of the key regulators of the antioxidant defence system, Nrf2 can mediate the expression of HO-1 and NQO1, thus playing an antioxidative stress role $(14,15)$. Civantos et al $(56)$ revealed that sitagliptin treatment could downregulate the expression of miR-200a, thereby abrogating its inhibitory effect on the Keap-1/Nrf2 pathway and ultimately alleviating diabetic nephropathy in rats. In STZ-induced diabetic mouse models, activated Nrf2 helped prevent the occurrence and progression of diabetic retinopathy (57). Moreover, existing research has reported that SIRT1 could activate the Nrf2/ARE pathway in glomerular mesangial cells (19). The present results revealed that allisartan isoproxil treatment could partially reverse the downregulation of both SIRT1 and Nrf2 protein expression in the heart tissue of diabetic rats. Moreover, the trend in the expression of HO-1 was consistent with that of Nrf2. The expression level of MDA 
A
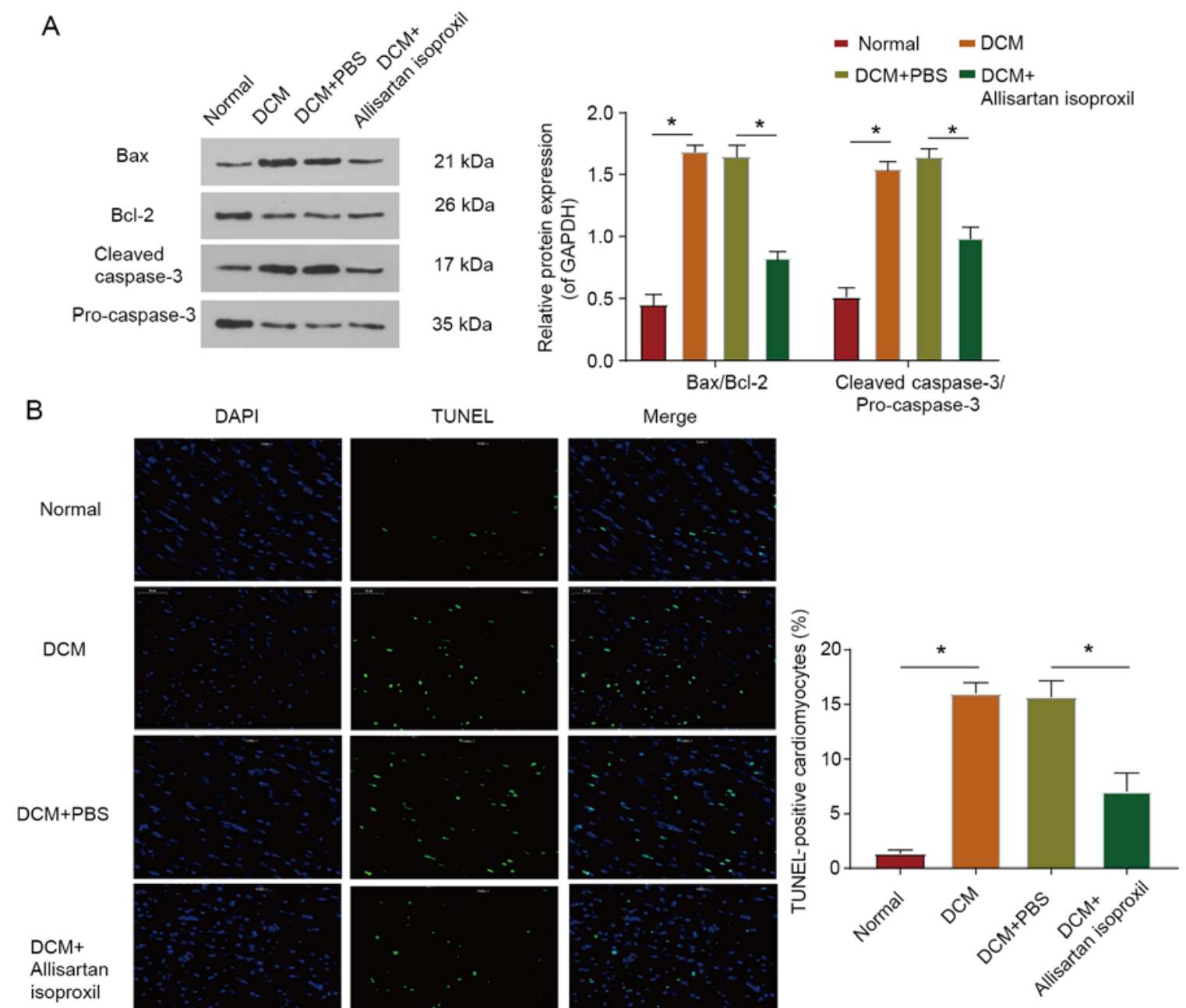

Figure 5. Cardiomyocyte apoptosis in DCM rats is reduced by allisartan isoproxil. (A) Cell apoptosis-related proteins Bax, Bcl-2 and caspase-3 and cleaved caspase-3 in heart tissues were analysed by immunoblotting. (B) Detection of apoptotic cells by TUNEL staining. Positive staining appears green; scale bar, $50 \mu \mathrm{m}$. The values are the mean $\pm \mathrm{SD}$ of $\mathrm{n}=6$ in each group. ${ }^{*} \mathrm{P}<0.05$. DCM, diabetic cardiomyopathy.

and the activity of SOD were also measured and the results demonstrated that allisartan isoproxil could reduce oxidative stress in DCM rats, at least in part, through the restoration of Nrf2.

The present results indicated that allisartan isoproxil alleviated DCM by attenuating diabetes-induced oxidative stress and inflammation through the SIRT1/Nrf2/NF- $\mathrm{KB}$ signalling pathway. In conclusion, allisartan isoproxil had a protective effect on DCM. Therefore, allisartan isoproxil may also be useful in the treatment of DCM.

\section{Acknowledgements}

Not applicable.

\section{Funding}

This study was supported by the Zhejiang Provincial Basic Public Welfare Research Projects (grant no. LGC20H020001), the Zhejiang Medical and Health Science and Technology Project (grant no. 2017KY200) and the Chinese Medicine Research Programme of Zhejiang Province (grant no. 2016ZB016).

\section{Availability of data and materials}

The datasets used and/or analysed during the present study are available from the corresponding author upon reasonable request.

\section{Authors' contributions}

XL, QJ and QZ contributed to the conception and design of the study. KW and MC performed the data analysis and interpretation of the data. QJ and QZ drafted the manuscript. XL and QJ performed critical revisions for important intellectual content of the manuscript. All authors approved the final version to be published.

\section{Ethics approval and consent to participate}

All animal study protocols were approved by the Institutional Ethics Committee of The First Affiliated Hospital with Nanjing Medical University (approval no. IACUC-1803019) and were performed according to the guidelines of the US Department of Health (NIH Publication no. 85-23, revised 1996) for the use and care of laboratory animals. This article does not contain any studies with human participants performed by any of the authors. 


\section{Patient consent for publication}

Not applicable.

\section{Competing of interests}

All authors declare that they have no competing interests.

\section{References}

1. Whiting DR, Guariguata L, Weil C and Shaw J: IDF diabetes atlas: Global estimates of the prevalence of diabetes for 2011 and 2030. Diabetes Res Clin Pract 94: 311-321, 2011.

2. Liu X, Xiao J, Zhu H, Wei X, Platt C, Damilano F, Xiao C, Bezzerides V, Boström P, Che L, et al: miR-222 is necessary for exercise-induced cardiac growth and protects against pathological cardiac remodeling. Cell Metab 21: 584-595, 2015.

3. Tribouilloy C, Rusinaru D, Mahjoub H, Tartière JM, Kesri-Tartière L, Godard S and Peltier M: Prognostic impact of diabetes mellitus in patients with heart failure and preserved ejection fraction: A prospective five-year study. Heart 94: 1450-1455, 2008.

4. Devereux RB, Roman MJ, Paranicas M, O'Grady MJ, Lee ET, Welty TK, Fabsitz RR, Robbins D, Rhoades ER and Howard BV: Impact of diabetes on cardiac structure and function: The strong heart study. Circulation 101: 2271-2276, 2000.

5. Howard BV, Cowan LD, Go O, Welty TK, Robbins DC and Lee ET: Adverse effects of diabetes on multiple cardiovascular disease risk factors in women. The Strong Heart Study. Diabetes Care 21: 1258-1265, 1998.

6. Ma ZG, Yuan YP, Xu SC, Wei WY, Xu CR, Zhang X, Wu QQ, Liao HH, Ni J and Tang QZ: CTRP3 attenuates cardiac dysfunction, inflammation, oxidative stress and cell death in diabetic cardiomyopathy in rats. Diabetologia 60: 1126-1137, 2017.

7. Zhou X, An G and Lu X: Hydrogen sulfide attenuates the development of diabetic cardiomyopathy. Clin Sci (Lond) 128: 325-335, 2015

8. Zhao MX, Zhou B, Ling L, Xiong XQ, Zhang F, Chen Q, Li YH, Kang YM and Zhu GQ: Salusin- $\beta$ contributes to oxidative stress and inflammation in diabetic cardiomyopathy. Cell Death Dis 8: e2690, 2017.

9. Rajesh M, Bátkai S, Kechrid M, Mukhopadhyay P, Lee WS, Horváth B, Holovac E, Cinar R, Liaudet L, Mackie K, et al Cannabinoid 1 receptor promotes cardiac dysfunction, oxidative stress, inflammation, and fibrosis in diabetic cardiomyopathy. Diabetes 61: 716-727, 2012.

10. Rajesh M, Mukhopadhyay P, Bátkai S, Patel V, Saito K, Matsumoto S, Kashiwaya Y, Horváth B, Mukhopadhyay B, Becker L, et al: Cannabidiol attenuates cardiac dysfunction, oxidative stress, fibrosis, and inflammatory and cell death signaling pathways in diabetic cardiomyopathy. J Am Coll Cardiol 56: 2115-2125, 2010.

11. Duecker R, Baer P, Eickmeier O, Strecker M, Kurz J, Schaible A, Henrich D, Zielen S and Schubert R: Oxidative stress-driven pulmonary inflammation and fibrosis in a mouse model of human ataxia-telangiectasia. Redox Biol 14: 645-655, 2018.

12. Rajasekaran NS, Varadharaj S, Khanderao GD, Davidson CJ, Kannan S, Firpo MA, Zweier JL and Benjamin IJ: Sustained activation of nuclear erythroid 2-related factor 2/antioxidant response element signaling promotes reductive stress in the human mutant protein aggregation cardiomyopathy in mice. Antioxid Redox Signal 14: 957-971, 2011.

13. Gu J, Cheng Y, Wu H, Kong L, Wang S, Xu Z, Zhang Z, Tan Y, Keller BB, Zhou H, et al: Metallothionein is downstream of $\mathrm{Nrf} 2$ and partially mediates sulforaphane prevention of diabetic cardiomyopathy. Diabetes 66: 529-542, 2017.

14. Lu MC, Ji JA, Jiang ZY and You QD: The Keap1-Nrf2-ARE pathway as a potential preventive and therapeutic target: An update. Med Res Rev 36: 924-963, 2016.

15. Magesh S, Chen Y and Hu L: Small molecule modulators of Keap1-Nrf2-ARE pathway as potential preventive and therapeutic agents. Med Res Rev 32: 687-726, 2012.

16. Zhao L, Qi Y, Xu L, Tao X, Han X, Yin L and Peng $\mathrm{J}$ : MicroRNA-140-5p aggravates doxorubicin-induced cardiotoxicity by promoting myocardial oxidative stress via targeting Nrf2 and Sirt2. Redox Biol 15: 284-296, 2018.
17. Karbasforooshan $\mathrm{H}$ and Karimi G: The role of SIRT1 in diabetic cardiomyopathy. Biomed Pharmacother 90: 386-392, 2017.

18. Guo R, Liu W, Liu B, Zhang B, Li W and Xu Y: SIRT1 suppresses cardiomyocyte apoptosis in diabetic cardiomyopathy: An insight into endoplasmic reticulum stress response mechanism. Int J Cardiol 191: 36-45, 2015.

19. Huang K, Huang J, Xie X, Wang S, Chen C, Shen X, Liu P and Huang H: Sirtl resists advanced glycation end products-induced expressions of fibronectin and TGF- $\beta 1$ by activating the Nrf2/ARE pathway in glomerular mesangial cells. Free Radic Biol Med 65: 528-540, 2013.

20. de Mingo Á, de Gregorio E, Moles A, Tarrats N, Tutusaus A, Colell A, Fernandez-Checa JC, Morales A and Marí M: Cysteine cathepsins control hepatic NF- $\kappa \mathrm{B}$-dependent inflammation via sirtuin-1 regulation. Cell Death Dis 7: e2464, 2016.

21. Dong B, Yu QT, Dai HY, Gao YY, Zhou ZL, Zhang L, Jiang H, Gao F, Li SY, Zhang YH, et al: Angiotensin-converting enzyme-2 overexpression improves left ventricular remodeling and function in a rat model of diabetic cardiomyopathy. J Am Coll Cardiol 59: 739-747, 2012.

22. Mehta PK and Griendling KK: Angiotensin II cell signaling: Physiological and pathological effects in the cardiovascular system. Am J Physiol Cell Physiol 292: C82-C97, 2007.

23. Mori J, Basu R, McLean BA, Das SK, Zhang L, Patel VB, Wagg CS, Kassiri Z, Lopaschuk GD and Oudit GY: Agonist-induced hypertrophy and diastolic dysfunction are associated with selective reduction in glucose oxidation: A metabolic contribution to heart failure with normal ejection fraction. Circ Heart Fail 5: 493-503, 2012.

24. Wu MY, Ma XJ, Yang C, Tao X, Liu AJ, Su DF and Liu JG: Effects of allisartan, a new AT(1) receptor blocker, on blood pressure and end-organ damage in hypertensive animals. Acta Pharmacol Sin 30: 307-313, 2009.

25. Zhang JQ, Yang GH, Zhou X, Liu JX, Shi R, Dong Y, Chen SB and Li YM: Effects of allisartan isoproxil on blood pressure and target organ injury in patients with mild to moderate essential hypertension. Medicine (Baltimore) 98: e14907, 2019.

26. Li Y, Li XH, Huang ZJ, Yang GP, Zhang GG, Zhao SP, Guo Y, Lu SJ, Ma JL, Meng FB, et al: A randomized, double blind, placebo-controlled, multicenter phase II trial of Allisartan Isoproxil in essential hypertensive population at low-medium risk. PLoS One 10: e0117560, 2015.

27. Os I, Franco V, Kjeldsen SE, Manhem K, Devereux RB, Gerdts E, Hille DA, Lyle PA, Okin PM, Dahlöf B and Oparil S: Effects of losartan in women with hypertension and left ventricular hypertrophy: Results from the Losartan Intervention for Endpoint Reduction in Hypertension Study. Hypertension 51: 1103-1108, 2008.

28. Bokma JP, Winter MM, van Dijk AP, Vliegen HW, van Melle JP, Meijboom FJ, Post MC, Berbee JK, Boekholdt SM, Groenink M, et al: Effect of losartan on right ventricular dysfunction: Results from the double-blind, randomized REDEFINE Trial (Right ventricular dysfunction in tetralogy of fallot: Inhibition of the renin-angiotensin-aldosterone system) in adults with repaired tetralogy of fallot. Circulation 137: 1463-1471, 2018.

29. Wang L, Li J and Li D: Losartan reduces myocardial interstitial fibrosis in DCMrats by inhibiting JAK/STAT signaling pathway. Int J Clin Exp Pathol 8: 466-473, 2015.

30. Axelsson A, Iversen K, Vejlstrup N, Ho CY, Havndrup O, Kofoed KF, Norsk J, Jensen M and Bundgaard H: Functional effects of losartan in hypertrophic cardiomyopathy-a randomised clinical trial. Heart 102: 285-291, 2016.

31. Shimada YJ, Passeri JJ, Baggish AL, O'Callaghan C, Lowry PA, Yannekis G, Abbara S, Ghoshhajra BB, Rothman RD, Ho CY, et al: Effects of losartan on left ventricular hypertrophy and fibrosis in patients with nonobstructive hypertrophic cardiomyopathy. JACC Heart Fail 1: 480-407, 2013.

32. Carswell CI and Goa KL: Losartan in diabetic nephropathy. Drugs 63: 407-416, 2003.

33. Ti Y, Xie GL, Wang ZH, Bi XL, Ding WY, Wang J, Jiang GH, $\mathrm{Bu}$ PL, Zhang Y, Zhong $\mathrm{M}$ and Zhang W: TRB3 gene silencing alleviates DCMin a type 2 diabetic rat model. Diabetes 60 : 2963-2974, 2011.

34. Bati K, Kwape TE and Chaturvedi P: Anti-Diabetic effects of an ethanol extract of cassia abbreviata stem bark on diabetic rats and possible mechanism of its action: -Anti-diabetic properties of cassia abbreviata. J Pharmacopuncture 20: 45-51, 2017.

35. Zheng D, Zhang Y, Hu Y, Guan J, Xu L, Xiao W, Zhong Q, Ren C, $\mathrm{Lu}$ J, Liang J and Hou J: Long noncoding RNA Crnde attenuates cardiac fibrosis via Smad3-Crnde negative feedback in diabetic cardiomyopathy. FEBS J 286: 1645-1655, 2019. 
36. Ghorbanzadeh V, Mohammadi M, Dariushnejad H, Chodari L and Mohaddes G: Effects of crocin and voluntary exercise, alone or combined, on heart VEGF-A and HOMA-IR of HFD/STZ induced type 2 diabetic rats. J Endocrinol Invest 39: 1179-1186, 2016.

37. Hou Y, Shao J, Fu Q, Li J, Sun J and He Z: Spray-dried nanocrystals for a highly hydrophobic drug: Increased drug loading, enhanced redispersity, and improved oral bioavailability. Int J Pharm 516: 372-379, 2017.

38. Livak KJ and Schmittgen TD: Analysis of relative gene expression data using real-time quantitative PCR and the 2(-Delta Delta C(T)) method. Methods 25: 402-408, 2001.

39. Bai Y, Cui W, Xin Y, Miao X, Barati MT, Zhang C, Chen Q, Tan Y, Cui T, Zheng Y and Cai L: Prevention by sulforaphane of DCMis associated with up-regulation of Nrf2 expression and transcription activation. J Mol Cell Cardiol 57: 82-95, 2013.

40. Koller WC and Vetere-Overfield B: Usefulness of a writing aid in writer's cramp. Neurology 39: 149-150, 1989.

41. Czarnecka AK, Milewski K and Zielińska M: Asymmetric dimethylarginine and hepatic encephalopathy: Cause, effect or association? Neurochem Res 42: 750-761, 2017.

42. Roul D and Recchia FA: Metabolic alterations induce oxidative stress in diabetic and failing hearts: Different pathways, same outcome. Antioxid Redox Signal 22: 1502-1514, 2015.

43. Palomer X,Pizarro-Delgado J and Vázquez-Carrera M: Emerging actors in diabetic cardiomyopathy: Heartbreaker biomarkers or therapeutic targets? Trends Pharmacol Sci 39: 452-467, 2018.

44. Zhu Y, Qian X, Li J, Lin X, Luo J, Huang J and Jin Z: Astragaloside-IV protects $\mathrm{H} 9 \mathrm{C} 2(2-1)$ cardiomyocytes from high glucose-induced injury via miR-34a-mediated autophagy pathway. Artif Cells Nanomed Biotechnol 47: 4172-4181, 2019.

45. Tsai TH, Lin CJ, Chua S, Chung SY, Chen SM, Lee CH and Hang CL: Deletion of RasGRF1 attenuated interstitial fibrosis in streptozotocin-induced DCMin mice through affecting inflammation and oxidative stress. Int J Mol Sci 19: 3094, 2018

46. Li L, Luo W, Qian Y, Zhu W, Qian J, Li J, Jin Y, Xu X and Liang G: Luteolin protects against DCMby inhibiting NF- $\kappa \mathrm{B}$-mediated inflammation and activating the Nrf2-mediated antioxidant responses. Phytomedicine 59: 152774, 2019.

47. Biernacka A, Cavalera M, Wang J, Russo I, Shinde A, Kong P, Gonzalez-Quesada C, Rai V, Dobaczewski M, Lee DW, et al: Smad3 signaling promotes fibrosis while preserving cardiac and aortic geometry in obese diabetic mice. Circ Heart Fail 8: 788-798, 2015

48. Gilmore TD: Introduction to NF-kappaB: Players, pathways, perspectives. Oncogene 25: 6680-6684, 2006.
49. Rojas A, Delgado-López F, González I, Pérez-Castro R, Romero J and Rojas I: The receptor for advanced glycation end-products: A complex signaling scenario for a promiscuous receptor. Cell Signal 25: 609-614, 2013

50. Lorenzo O, Picatoste B, Ares-Carrasco S, Ramírez E, Egido J and Tuñón J: Potential role of nuclear factor $\kappa \mathrm{B}$ in diabetic cardiomyopathy. Mediators Inflamm 2011: 652097, 2011.

51. Li H, Shi Y, Wang X, Li P, Zhang S, Wu T, Yan Y, Zhan Y, Ren Y, Rong X, et al: Piceatannol alleviates inflammation and oxidative stress via modulation of the Nrf2/HO-1 and NF- $\kappa$ B pathways in diabetic cardiomyopathy. Chem Biol Interact 310: 108754, 2019.

52. Westermann D, Rutschow S, Van Linthout S, Linderer A, Bücker-Gärtner C, Sobirey M, Riad A, Pauschinger M, Schultheiss HP and Tschöpe C: Inhibition of p38 mitogen-activated protein kinase attenuates left ventricular dysfunction by mediating pro-inflammatory cardiac cytokine levels in a mouse model of diabetes mellitus. Diabetologia 49: 2507-2513, 2006.

53. Westermann D, Rutschow S, Jäger S, Linderer A, Anker S, Riad A, Unger T, Schultheiss HP, Pauschinger M and Tschöpe C: Contributions of inflammation and cardiac matrix metalloproteinase activity to cardiac failure in diabetic cardiomyopathy: The role of angiotensin type 1 receptor antagonism. Diabetes 56: 641-646, 2007.

54. Yu T, Sheu SS, Robotham JL and Yoon Y: Mitochondrial fission mediates high glucose-induced cell death through elevated production of reactive oxygen species. Cardiovasc Res 79: 341-351, 2008.

55. Guo Y, Zhuang X, Huang Z, Zou J, Yang D, Hu X, Du Z, Wang L and Liao X: Klotho protects the heart from hyperglycemia-induced injury by inactivating ROS and NF- $\mathrm{B}$-mediated inflammation both in vitro and in vivo. Biochim Biophys Acta Mol Basis Dis 1864: 238-251, 2018.

56. Civantos E, Bosch E, Ramirez E, Zhenyukh O, Egido J, Lorenzo O and Mas S: Sitagliptin ameliorates oxidative stress in experimental diabetic nephropathy by diminishing the miR-200a/Keap-1/Nrf2 antioxidant pathway. Diabetes Metab Syndr Obes 10: 207-222, 2017.

57. Xu Z, Wei Y, Gong J, Cho H, Park JK, Sung ER, Huang H, Wu L, Eberhart C, Handa JT, et al: NRF2 plays a protective role in diabetic retinopathy in mice. Diabetologia 57: 204-213, 2014.

This work is licensed under a Creative Commons Attribution-NonCommercial-NoDerivatives 4.0 International (CC BY-NC-ND 4.0) License. 